\title{
Efficacy Comparison of Three Kinds of Distal Tibial Hemiepiphyseal Implants in the Treatment of Postoperative Ankle Valgus of Congenital Pseudarthrosis of the Tibia
}

\author{
Xiongke Hu \\ University of South China \\ Anping Li \\ University of South China \\ Kun Liu \\ University of South China \\ Haibo Mei ( $\square$ meihaibo1@sina.com ) \\ University of South China
}

\section{Research Article}

Keywords: Ankle Valgus, Hemiepiphyseal, Trans-epiphyseal Plate Screws, "U”-shaped Screws, Congenital Pseudarthrosis of the Tibia, Evidence level, $\otimes$ Retrospective Study

Posted Date: October 5th, 2021

DOI: https://doi.org/10.21203/rs.3.rs-936575/v1

License: (9) This work is licensed under a Creative Commons Attribution 4.0 International License.

Read Full License 


\section{Abstract}

Background: Congenital pseudarthrosis of the tibia is a complex and serious disease in orthopedics which often requires multiple operations for treatment. Postoperative ankle valgus deformity is easily seen after the operation of congenital pseudarthrosis of the tibia. The aim of this study is to retrospectively evaluate the safety of three different implants for treating postoperative ankle valgus after congenital pseudarthrosis of the tibia.

Methods: A total of 41 patients with postoperative ankle valgus after congenital pseudarthrosis of the tibia from December 2010 to July 2019 were selected. Out of these 41 patients, 23 patients were treated with " $U$ "-shaped tension screw, 10 patients were treated with hollow screw and 8 patients were treated with cortical bone screw. The evaluation index was tibiotalar angle. The general data, preoperative, postoperative and final follow-up imaging data were recorded, and the deformity correction rate and complications were compared.

Results: all the patients were performed with postoperative follow-up visit for at least 12 months (31 months on average). In the " $U$ "-shaped tension screw group, the preoperative tibiotalar angle was $\left(74.8 \pm 4.8^{\circ}\right)$, the tibiotalar angle was $\left(85.8 \pm 4.5^{\circ}\right)$ when the internal fixation was removed; in the hollow screw group, the average preoperative tibiotalar angle was $\left(72.2 \pm 6.1^{\circ}\right)$, the average tibiotalar angle was $\left(88.4 \pm 5.1^{\circ}\right)$ when the internal fixation was removed; in the cortical bone screw group, the average preoperative tibiotalar angle was $\left(75.1 \pm 4.2^{\circ}\right)$, the average tibiotalar angle was $\left(88.4 \pm 5.1^{\circ}\right)$ when the internal fixation was removed. The correction effect of the " $U$ "-shaped tension screw group was better than that of the other two groups, but the difference was not significant (the correction rate of the " $\mathrm{U}$ "shaped tension screw group was $0.71^{\circ}$ month, with that of in the hollow screw group and cortical bone screw group being $0.64^{\circ}$ /month and $0.61 \%$ month respectively, $\left.P \otimes 0.05\right)$. One case of internal fixation complication was reported in the hollow screw group; two cases of missing correction effect were reported, one in cortical bone screw group and one in hollow screw group; and two cases showing symptom of wound pain were reported in the "U"-shaped screw group.

Conclusion: Ankle valgus is a common postoperative complication of congenital tibial pseudarthrosis. Temporary hemiepiphyseal is an effective treatment for postoperative ankle valgus deformity of congenital pseudarthrosis of the tibia in children. Through comparison, the "U"-shaped tension screw provides relatively better orthopedic results and has a lower rate of internal fixation complications.

\section{Background}

As a kind of tibial deformity caused by the abnormal development of tibia, congenital pseudarthrosis of the tibia (CPT) is often manifested as tibial medullary cavity stenosis, angulation deformity or bone nonunion, etc., which eventually leads to nonunion of the pseudarthrosis [1-2]. At present, the etiology of CPT is still unclear. Therefore, the treatment of CPT has always been one of the most challenging diseases in pediatric orthopedics due to the difficulties of bone fusion, persistent angulation and serious 
limb inequality [1-2]. Currently, our hospital is the medical unit with the largest number of patients suffering from CPT in China. It is found that comprehensive surgical treatment, such as excision of diseased periosteum and pseudarthrosis, intramedullary nail fixation of foot and ankle, encapsulated autogenous iliac bone graft, llizarov external fixation, etc., can significantly improve the fracture fusion rate and reduce the rate of recurrent fracture [3-6]. However, in the follow-up treatment of CPT, one of the problems that should be paid enough attention to is the residual valgus deformity of ankle. After 4.3 years of long-term follow-up, Thabet et al. found that the incidence of postoperative ankle valgus after CPT was as high as 35\% [7]. From August 2008 to September 2018, a total of 231 patients with CPT were treated by combined surgery, of which 58 patients had postoperative ankle valgus, with an incidence of $25.1 \%$.

Children's ankle valgus can cause difficulties in wearing shoes, walking instability or mechanical pain and other symptoms. Generally, conservative treatment of ankle valgus come with poor effect, so surgical intervention is needed $[8,9]$. Presently, the commonly used methods of ankle reconstruction mainly include medial hemiepiphyseal and supramalleolar osteotomy [8-10]. Osteotomy permits rapid correction, but it has the disadvantages of long operation time, great trauma and more complications [11]. Temporary hemiepiphyseal has been proved to be an effective treatment for patients with immature bone. It can gradually correct the coronal deformity of ankle joint by temporarily blocking the growth of one side of epiphyseal plate $[9,12]$. At present, tension band screws and trans-epiphyseal plate screws are commonly used in temporary hemiepiphyseal fixation $[9,12,13]$, but there is still a lack of large sample data to compare the correction rate and complications of postoperative ankle valgus of CPT with different implants.

Therefore, this study is to compare the efficacy of three different internal fixation implants in the treatment of postoperative ankle valgus of CPT, especially the rate of deformity correction and the incidence of complications associated with each implant.

\section{Methods}

After the approval of the ethics committee of hospital, we conducted a retrospective study on children with postoperative ankle valgus of CPT from December 2010 to July 2019. All research on the patients complied with the Declaration of Helsinki and obtained the consent of the legal guardian. Inclusive criteria: $\triangle$ Patients who were diagnosed with CPT and treated with periostectomy + encapsulated bone graft + fixation, and with postoperative ankle valgus deformity; $\otimes$ Patients who was 2-14 years old, with immature skeleton; $\mathbb{X}$ The $X$-ray film of both lower limbs in standing position indicated the existence of ankle valgus deformity; $\otimes$ The observation index was tibiotalar angle. Exclusion criteria: $\otimes$ Patients with ankle valgus deformity caused by traumatic epiphyseal plate premature closure, epiphyseal plate lesion after infection and other reasons; $\otimes$ Patients with sagittal deformity; $\triangle$ Patients who were not conducted with follow-up.

\section{General data}


According to the above criteria, a total of 41 patients were included in the study, of which 24 were male and 17 were female. The data of the patients included the ages when they started to receive the operation, the time of hemiepiphyseal block, the preoperative and postoperative tibial angle (Table 1). Full-length X-ray films of double lower limbs with load were taken for all the patients, and the degree of ankle valgus was evaluated by measuring the tibiotalar angle. As tibia bending often occurs in patients with CPT, we choose the angle between the articular surface of talar fornix and the midpoint line crossing the center of tibial intercondylar ridge and the level of ankle joint space and (Fig.1).

\section{Surgical operation}

The patients were administrated with antibiotics for preoperative prevention, given general anesthesia in the supine position, and appropriate pneumatic tourniquet was selected according to their weight. Before operation, the position of medial epiphyseal plate of distal tibia was determined by $\mathrm{C}$-arm fluoroscopy. A skin incision was made on the medial side of the distal tibia, about $4 \mathrm{~cm}$ in length, exposing to the periosteum layer by layer to avoid injury of blood vessels and nerves. According to the thickness of tibia and the severity of ankle valgus, " $U$ "-shaped tension screws with different lengths and angles were selected. Otherwise, cortical bone screws or hollow screws were screwed in from distal tibial epiphysis to proximal tibial metaphysis. Anteroposterior and lateral X-ray images obtained by intraoperative fluoroscopy were used to check the position of the implant. Then the wound was rinsed, the tourniquet was deflated, the wound was compressed and electrocautery was used to stop bleeding. The antiadhesion film was placed on the wound to prevent postoperative scar adhesion, and the absorbable suture was used to suture the wound.

\section{Follow up and removal of internal fixation}

The patients were reexamined every 3-4 months after the operation, and the X-ray films of both lower limbs when standing were taken to monitor the limb growth and deformity correction. The tibiotalar angle was recorded every time. In our study, deformity correction was achieved after 6-30 months for " $\mathrm{U}$ "shaped tension screw implantation, compared with 8-36 months for hollow screws and 17-27 months for cortical screws. Based on our experience, the valgus deformity can be slightly overcorrected because some rebound may occur after the implant is removed. After removal of internal fixation, the patients were conducted with plaster fixation for 4-6 weeks, and then all activities were restored. All the patients were performed with reexamination every 6 months until the bone matured, and the recurrence of deformity was monitored.

\section{Results}

All operations were performed by the same group of doctors, with the operation time of 20-70 minutes (42 minutes on average). In the " $U$ "-shaped tension screw group, the preoperative tibiotalar angle was $\left(74.8 \pm 4.8^{\circ}\right)$, the tibiotalar angle was $\left(85.8 \pm 4.5^{\circ}\right)$ when the internal fixation was removed; in the hollow screw group, the average preoperative tibiotalar angle was $\left(72.2 \pm 6.1^{\circ}\right)$, the average tibiotalar angle was $\left(88.4 \pm 5.1^{\circ}\right)$ when the internal fixation was removed; in the cortical bone screw group, the average 
preoperative tibiotalar angle was $\left(75.1 \pm 4.2^{\circ}\right)$, the average tibiotalar angle was $\left(88.4 \pm 5.1^{\circ}\right)$ when the internal fixation was removed (Table 2) (Fig.2). The correction rate of the " $U$ "-shaped tension screw group was $0.71 \%$ month, and that of in the hollow screw group and cortical bone screw group was $0.64 \%$ month and $0.61 \%$ month respectively. In the hollow screw group, there was one case of internal fixation complication, and the screw was embedded in the bone, so that it was difficult to take out the internal fixation (Fig.3). Correction effect was lost for 1 patient in the cortical bone screw group and 1 patient in the hollow screw group, and 2 cases of symptom of wound pain occurred in the " $U$ "-shaped tension screw group. No complications such as wound infection occurred in any patient. At the last follow-up, the ankle joint activity of all patients was normal.

\section{Discussion}

$\mathrm{CPT}$ is a troublesome disease in pediatric orthopedics because of segmental skeletal dysplasia, and is often accompanied by nonunion and tibial deformity. At present, the commonly used surgical treatment methods mainly include intramedullary nail fixation, llizarov external fixation, combination of intramedullary nail and llizarov external fixation, as well as vascularized fibula transplantation $[14,15]$. Because vascularized fibula transplantation tends to form pseudarthrosis, and single internal fixation or external fixation cannot ensure stability to help fracture healing, combination of intramedullary nail and Ilizarov external fixation are preferred clinically in order to obtain a higher fracture fusion rate. 15 patients with CPT were treated by Agashe et al. with combination of intramedullary nail and llizarov external fixation, of which, 14 patients had bony union, while 7 patients suffered from ankle valgus deformity [16]. A total of 56 patients with CPT were treated by our hospital in early years by intramedullary nail combined with encapsulated bone graft and llizarov external fixation. After long-term follow-up with an average of 8.5 years, it was found that the fracture healing rate reached $89.2 \%$, but with the incidence of ankle valgus deformity of $17.9 \%$ [4]. The patients with CPT are prone to the proximal migration of distal fibula and ankle valgus deformity after ankle intramedullary nailing. Therefore, it is recommended to closely monitor the ankle function in the long-term treatment of patients with CPT.

As early as the 1940s, Phemister first proposed the idea of treating children's bone deformity by epiphyseal plate block [17]. Subsequently, Stevens further proposed the concept of "guiding growth", that is to restrain the growth of epiphyseal plate of one side by internal fixation under engineering mechanics, and to retain the normal growth of epiphyseal plate of the opposite side, so as to correct bone angulation $[9,18]$. Currently, the commonly-used internal fixation materials for guiding growth mainly include " 8 " or "U"-shaped tension band screws and epiphyseal plate screws $[13,19]$. We conducted an experiment by using " $U$ "-shaped tension band screws, cortical bone screws and hollow screws respectively in 41 patients with postoperative ankle valgus deformity of CPT from December 2010 to July 2019. It was found that the operation was safe and simple, and there was no need for fixation and load limitation. The results showed that both "U"-shaped tension screws and epiphyseal plate screws could gradually correct ankle valgus deformity in patients with immature bone. The comparison of the three kinds of internal fixation indicated that the " $U$ "-shaped tension screw could correct at a faster speed $(0.71 \%$ month), compared with hollow screws $\left(0.64^{\circ}\right.$ month) and cortical bone screws $(0.61 \%$ month). But there was no 
statistical difference. Furthermore, comparing the age of the patients in the three groups, it was found that the average age of the patients in the "U"-shaped tension screw group was the smallest. The study suggests that the correction of ankle valgus deformity is significantly related to the age of patients, and young patients often have better correction improvement [9,20]. Dirscoll et al. [21] reported that a total of 42 patients with the average age of 10.3 years who suffered from ankle valgus deformity were treated with epiphyseal screws and tension band screws. They found that the average correction rate of epiphyseal screws was $0.55^{\circ} /$ month, the average correction rate of tension band screws was $0.36 \%$ month. Because of the early onset of CPT, ankle valgus deformity occurs at a young age after surgical treatment. The average age of " $U$ "-shaped tension screw group was 7.04 years old, and that of hollow screw group and cortical bone screw group was 7.46 years old and 7.33 years old respectively. Therefore, the correction effect was better than that reported by other scholars.

In this study, the self-developed " $U$ "-shaped tension screws were selected, which is an improved version of the widely used Blount-staples. The head of the screw has three shallow indentations, and the transverse part and the longitudinal axis form $24-30^{\circ}$ angle. It has the advantages of close fitting the tibia and strong holding force. The direction of " $U$ "-shaped tension screw implantation is required to be parallel to the growth plate to ensure that the depth of implantation is about half of the diameter of the tibia. At the same time, the "U"-shaped screws with appropriate angle are selected, and the shorter side is implanted into the proximal, so as to fit the medial cortical bone better. As for the position of the epiphyseal screws, it is recommended that it should be implanted vertically to the growth plate as far as possible, and placed in the middle of the medial malleolus, while it is noted that it should not be inserted into the joint cavity. After the removal of internal fixation, there was no injury of epiphyseal plate which affected the growth of limbs for all the patients.

The ages and growth rate of different patients are not consistent, so it is difficult to accurately predict the individual correction rate. Therefore, close imaging follow-up is necessary for the patients to receive distal tibial hemiepiphyseal, which should be continued until bone maturity. Hemiepiphyseal with epiphyseal plate screws can lead to complications such as over-correction or screw buried by bone [19,22]. Out of the 18 patients who were performed with epiphyseal plate screw block, 1 patient had the complication of screw ingrowth into the bone, and 1 patient with cortical bone screws and 1 patient with hollow screws had the loss of correction effect after removal of internal fixation. The tibiotalar angles of the 2 patients with the loss of correction effect were greater than $80^{\circ}$, which could be corrected by the range of podarthrum motion. " 8 "-shaped tension band plate combined with screw fixation is also the most commonly used hemiepiphyseal material, which is commonly used in the distal femur and proximal tibia. Considering less soft tissue and lack of muscle tissue coverage in the medial ankle, the " $U$ "-shaped tension screws with lower notch were selected. Previously, the Blount-staples were often used to be inserted into the epiphysis of children who were not fully ossified, and with their growth, staples could withdraw $[13,23]$. However, due to the increase of the screw width and the indentations of the head, there was no screw withdrawal phenomenon in all 23 patients treated with " $U$ "-shaped tension screw. At the same time, additional concern was required on the wound infection of the patients treated with tension band screws, especially the thinner patients. There was no wound infection in any of our patients treated 
with "U"-shaped tension screws, but there were 2 cases with obvious postoperative pain in the internal fixation position, and the symptoms disappeared after oral medication and physical therapy.

Temporary hemiepiphyseal by using cortical bone screws, "U"-shaped tension screws, hollow screws, etc., is an effective treatment for postoperative ankle valgus deformity in patients with CPT. At the same time, the "U"-shaped tension screw provides relatively better orthopedic results and has a lower rate of internal fixation complications. However, our study has some limitations. The effect of different age groups on the correction rate was not quantified. Subsequently, more strict grouping and longer-term follow-up should be set up.

\section{Declarations}

Ethics approval and consent to participate]Ethical approval was obtained from the Hunan Children's Hospital Ethics Committee. Written informed consent was obtained from individual or guardian participants.

\section{Consent to Publish:}

The Author confirms:

- that the work described has not been published before.

- All authors agree to publication in the Journal indicated below.

Authors Contributions: Hu X and Mei H carried out the majority of the study, analyzed data and prepared the manuscript; Hu K and Li A supervised the study and wrote the first draft of manuscript; Li A and Liu K assisted with the study and the analysis of the data. All authors approved the final manuscript.

Funding: This work was supported by the Natural Science Foundation of Hunan Province, China (Grant No. 2020JJ5900), the Clinical Research Center for Limb Deformity of Children in Hunan Province $\mathbb{G r a n t}$ No. 2019SK4006)

Competing interests: No conflict of interest.

Availability of data and materials[The data sets supporting the results of this article are included within the article and its additional files.

\section{References}

1. Pannier, S. Congenital Pseudarthrosis of the Tibia. Orthop Traumatol Surg Res, 97, 750-761 (2011).

2. Paley, D. Congenital Pseudarthrosis of the Tibia: Biological and Biomechanical Considerations to Achieve Union and Prevent Refracture. J Child Orthop, 13, 120-133 (2019). 
3. Zhu, G. H., Mei, H. B. \& He, R. G. etc. Effect of Distraction Osteogenesis in Patient with Tibial Shortening after Initial Union of Congenital Pseudarthrosis of the Tibia (CPT): a Preliminary Study. BMC Musculoskelet Disord, 16, 216 (2015).

4. Zhu, G. H., Mei, H. B. \& He, R. G. etc. Combination of Intramedullary Rod, Wrapping Bone Grafting and Ilizarov's Fixator for the Treatment of Crawford type IV Congenital Pseudarthrosis of the Tibia: Midterm Follow up of 56 cases. BMC Musculoskelet Disord 2016, 17:443.

5. Liu, Y. X., Mei, H. B. \& Zhu, G. H. ; etc. Congenital Pseudarthrosis of the Tibia in Children: Should We Defer Surgery Until 3 years old?. J Pediatr Orthop B 2017,27:17-25.

6. Liu, Y. X., Yang, G. \& Liu, K. etc. Combined Surgery with 3-in-1 Osteosynthesis in Congenital Pseudarthrosis of the Tibia with Intact Fibula. Orphanet J Rare Dis 2020, 15:62.

7. Thabet, A. M., Paley, D. \& Kocaoglu, M. etc. Periosteal grafting for congenital pseudarthrosis of the tibia: a preliminary report. Clin Orthop Relat Res, 466, 2981-2994 (2008).

8. Chang, F. M., Ma, J. \& Pan, Z. etc. Rate of Correction and Recurrence of Ankle Valgus in Children Using a Transphyseal Medial Malleolar Screw. J Pediatr Orthop 2015, 35:589-592.

9. Stevens, P. M., Kennedy, J. M. \& Hung, M. Guided Growth for Ankle Valgus. J Pediatr Orthop, 31, 878883 (2011).

10. Davids, J. R. The Foot and Ankle in Cerebral Palsy. Orthop Clin North Am, 41, 579-593 (2010).

11. Payman, K. R., Patenall, V. \& Borden, P. etc. Complications of Tibial Osteotomies in Children with Comorbidities. J Pediatr Orthop, 22, 642-644 (2002).

12. Gottliebsen, M., Shiguetomi-Medina, J. M. \& Rahbek, O. etc. Guided Growth: Mechanism and Reversibility of Modulation. J Child Orthop 2016, 10: 471-477.

13. Burghardt, R. D., Herzenberg, J. E. \& Standard, S. C. ; etc. Temporary Hemiepiphyseal Arrest using a Screw and Plate Device to Treat Knee and Ankle Deformities in Children: a Preliminary Report.J Child Orthop2008, (3):187-197.

14. Paley, D. Congenital Pseudarthrosis of the Tibia: Biological and Biomechanical Considerations to Achieve Union and Prevent Refracture. J Child Orthop, 13, 120-133 (2019).

15. Borzunov, D. Y., Chevardin, A. Y. \& Mitrofanov, A. I. Management of Congenital Pseudarthrosis of the Tibia with the llizarov Method in a Paediatric Population: Influence of Aetiological Factors. Int Orthop, 40, 331-339 (2016).

16. Agashe, M. V., Song, S. H. \& Refai, M. A. etc. Congenital Pseudarthrosis of the Tibia Treated with a Combination of Ilizarov's Technique and Intramedullary Rodding. Acta Orthop, 83, 515-522 (2012).

17. Phemister, D. B. Operative Arrestment of Longitudinal Growth of Bone in the Treatment of Deformity. J Bone Joint Surg Am, 15 (79), 777-792 (1933).

18. Stevens, P. M. Guided Growth for Angular Correction: a Preliminary Series using a Tension Band Plate. J Pediatr Orthop, 27, 253-259 (2007).

19. Aurégan, J. C., Finidori, G. \& Cadilhac, C. etc. Children Ankle Valgus Deformity Treatment using a Transphyseal Medial Malleolar screw. Orthop Traumatol Surg Res, 97, 406-409 (2011). 
20. Oosterbos, M. V., Zwan, A. \& Woude, H. etc. Correction of Ankle Valgus by Hemiepiphysiodesis using the Tension Band Principle in Patients with Multiple Hereditary Exostosis. J Child Orthop, 10, 267273 (2016).

21. Driscoll, M. D., Linton, J. \& Sullivan, E. etc. Medial Malleolar Screw Versus Tension-band Plate Hemiepiphysiodesis for Ankle Valgus in the Skeletally Immature. J Pediatr Orthop, 34, 441-446 (2013).

22. Davids, J. R., Valadie, A. L. \& Ferguson, R. L. etc. Surgical Management of Ankle Valgus in Children: use of a Transphyseal Medial Malleolar Screw. J Pediatr Orthop, 17, 3-8 (1997).

23. Mielke, C. H. \& Stevens, P. M. Hemiepiphyseal Stapling for Knee Deformities in Children Younger than 10 years: a Preliminary Report. J Pediatr Orthop, 16, 423-429 (1996).

\section{Tables}

Table 1 Patient data

\begin{tabular}{|c|c|c|c|c|c|}
\hline & \multirow{2}{*}{$\begin{array}{l}\text { Age at surgery } \\
\text { (years) }\end{array}$} & \multirow{2}{*}{$\begin{array}{l}\text { Time of } \\
\text { hemiepiphyseal } \\
\text { (months) }\end{array}$} & \multicolumn{3}{|c|}{ Tibiotalar angle $\left({ }^{\circ}\right)$} \\
\hline & & & Preoperative & Postoperative & $\begin{array}{l}\text { Last } \\
\text { follow } \\
\text { up }\end{array}$ \\
\hline "U” screw & $7.04 \pm 2.63$ & $15.65 \pm 6.2$ & $74.8 \pm 4.8$ & $85.8 \pm 4.5$ & $84.3 \pm 5.2$ \\
\hline $\begin{array}{l}\text { Hollow } \\
\text { screw }\end{array}$ & $7.46 \pm 3.07$ & $24.9 \pm 9.17$ & $72.2 \pm 6.1$ & $86.3 \pm 5.8$ & $83.4 \pm 7.0$ \\
\hline $\begin{array}{l}\text { Cortical } \\
\text { screw }\end{array}$ & $7.33 \pm 1.28$ & $22.25 \pm 4.04$ & $75.1 \pm 4.2$ & $88.4 \pm 5.1$ & $84.4 \pm 8.1$ \\
\hline
\end{tabular}

Table 2 The corresponding changes of tibiotalar angle in each patient 


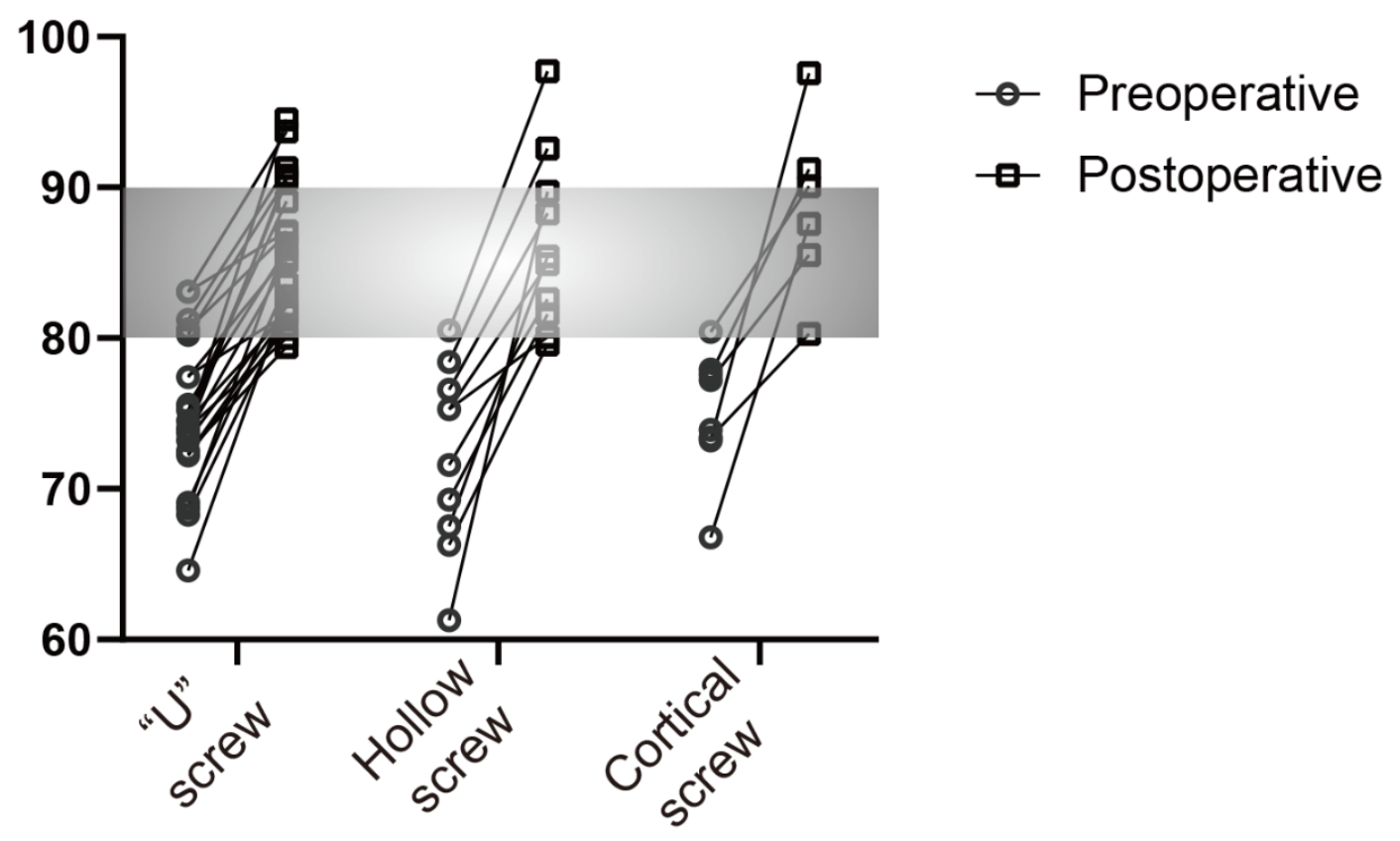

Figures 


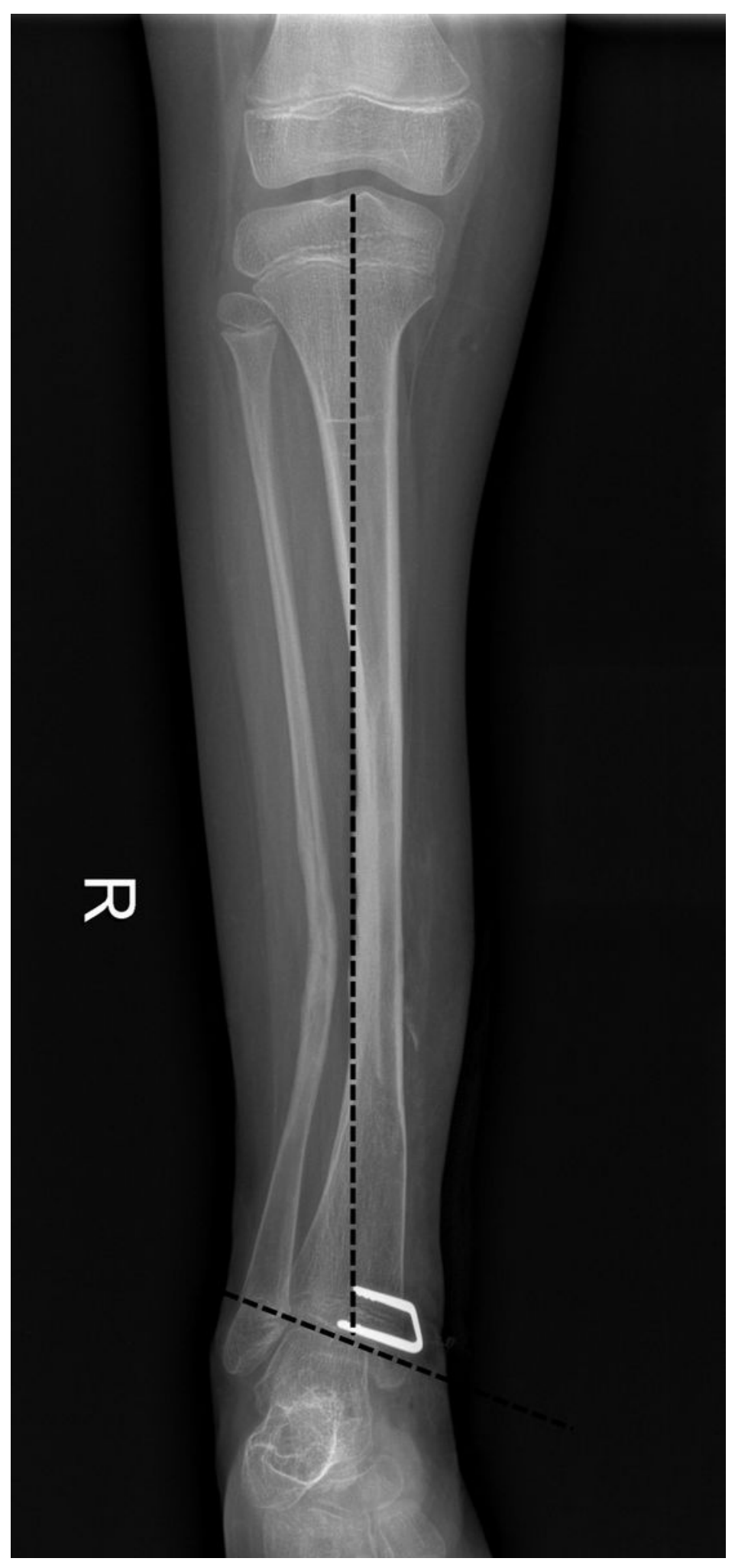

\section{Figure 1}

Tibiotalar angle: we choose the angle between the middle line between the center of tibial intercondylar eminence and the level of ankle joint space and the articular surface of talar fornix. 

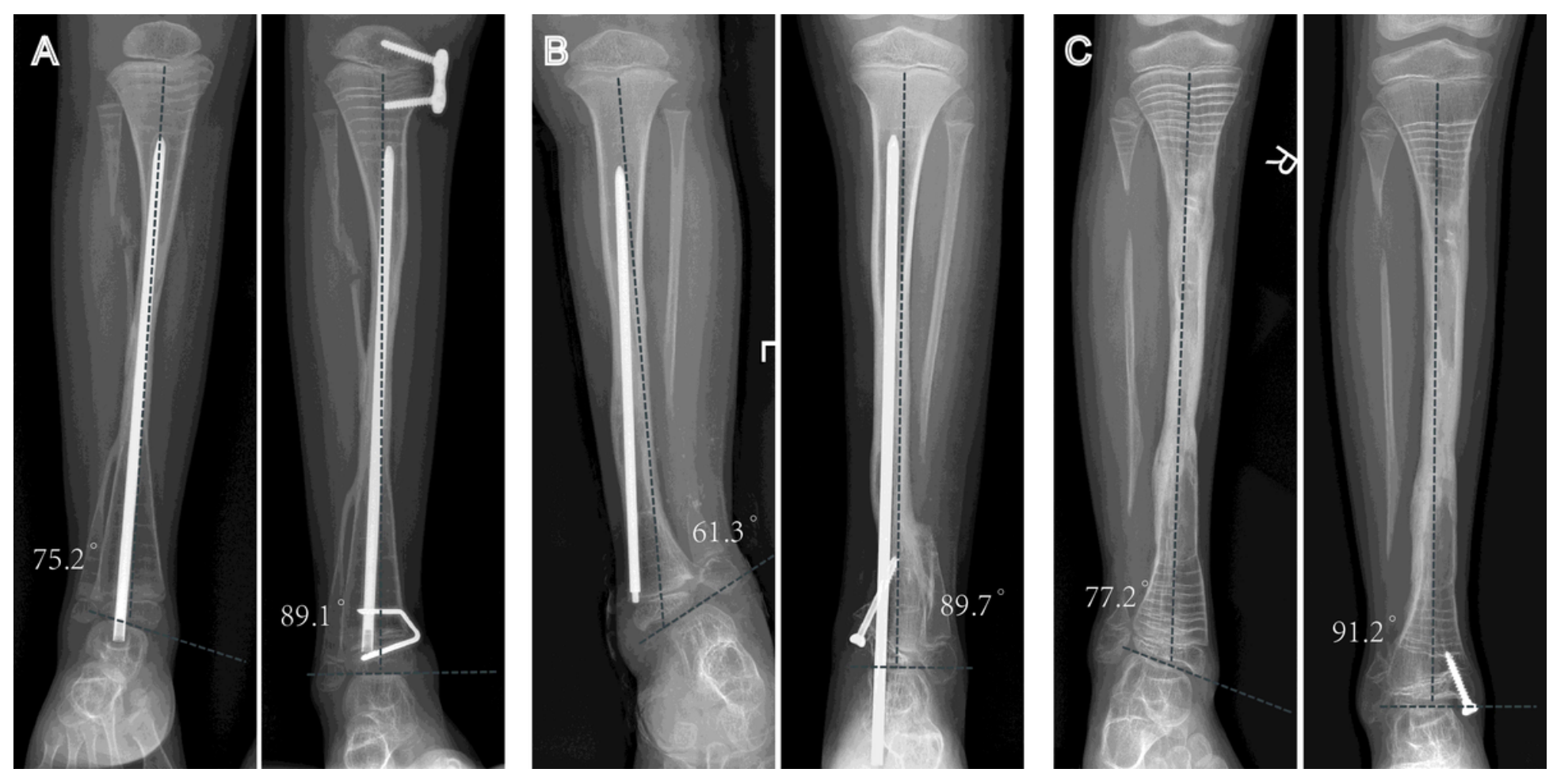

Figure 2

Typical cases with the correction of ankle valgus deformit. A. A 5-year-old boy presented with ankle valgus deformity after $\mathrm{CPT}$, with the preoperative tibiotalar angle of $75.2^{\circ}$. He was treated with hemiepiphyseal plate block with the "U"-shaped tension screws for 12 months, and the correction effect was satisfactory; B. A 7-year-old girl with preoperative ankle valgus deformity, with the tibiotalar angle of $61.3^{\circ}$. She was treated with hemiepiphyseal plate block with hollow screws, then the ankle valgus deformity was corrected; C. A 8-year-old boy with preoperative ankle valgus deformity with the tibiotalar angle of of $77.2^{\circ}$. After 19 months of treatment with medial-malleolus hemiepiphyseal plate block with cortical bone screws, the deformity was gradually corrected. 

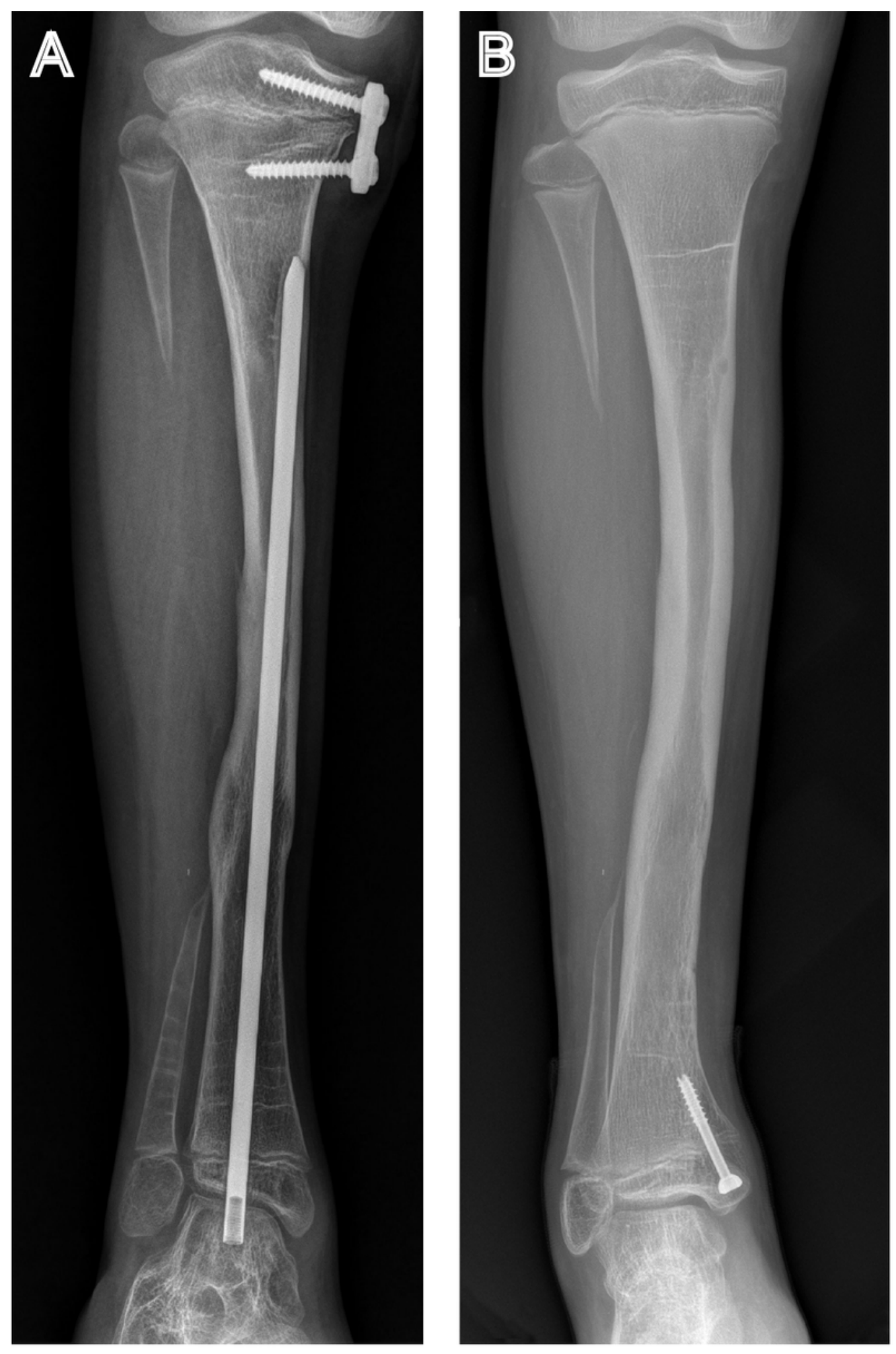

\section{Figure 3}

There was one patient with internal fixation complication in the hollow screw group. Angular deformity of the left leg for the child patient was found shortly after birth, and was diagnosed with pseudarthrosis of tibia. At the age of 5 years, the patient underwent periostectomy + encapsulated bone graft + fixation. The patient developed ankle valgus deformity 4 years after the operation (A). The ankle valgus deformity was 
basically corrected after 42 months of hemiepiphyseal block with hollow screws, but the screw heads was buried in the medial malleolus, so that it is difficult to remove the internal fixation (B). 